\title{
Advancements in detection of SARS-CoV-2 infection for confronting COVID-19 pandemics
}

\author{
Yuan Zhou', Li Zhang ${ }^{1}$, You-Hua Xie ${ }^{1,2 \bowtie}$ and Jian Wu (iD ${ }^{1,3,4 \bowtie}$ \\ (C) The Author(s), under exclusive licence to United States and Canadian Academy of Pathology 2021
}

\begin{abstract}
As one of the major approaches in combating the COVID-19 pandemics, the availability of specific and reliable assays for the SARSCoV-2 viral genome and its proteins is essential to identify the infection in suspected populations, make diagnoses in symptomatic or asymptomatic individuals, and determine clearance of the virus after the infection. For these purposes, use of the quantitative reverse transcriptase polymerase chain reaction (qRT-PCR) for detection of the viral nucleic acid remains the most valuable in terms of its specificity, fast turn-around, high-throughput capacity, and reliability. It is critical to update the sequences of primers and probes to ensure the detection of newly emerged variants. Various assays for increased levels of lgG or lgM antibodies are available for detecting ongoing or past infection, vaccination responses, and persistence and for identifying high titers of neutralizing antibodies in recovered individuals. Viral genome sequencing is increasingly used for tracing infectious sources, monitoring mutations, and subtype classification and is less valuable in diagnosis because of its capacity and high cost. Nanopore target sequencing with portable options is available for a quick process for sequencing data. Emerging CRISPR-Cas-based assays, such as SHERLOCK and AIOD-CRISPR, for viral genome detection may offer options for prompt and point-of-care detection. Moreover, aptamer-based probes may be multifaceted for developing portable and high-throughput assays with fluorescent or chemiluminescent probes for viral proteins. In conclusion, assays are available for viral genome and protein detection, and the selection of specific assays depends on the purposes of prevention, diagnosis and pandemic control, or monitoring of vaccination efficacy.
\end{abstract}

Laboratory Investigation (2022) 102:4-13; https://doi.org/10.1038/s41374-021-00663-w

\section{INTRODUCTION}

According to the World Health Organization (WHO), cases of pneumonia of unknown etiology were reported during late 2019 and early 2020 in several regions. This pneumonia was later named coronavirus infectious disease $\left(\right.$ COVID-19) ${ }^{1}$, and its pathogen was identified as severe acute respiratory syndrome coronavirus 2 (SARS-CoV-2) ${ }^{2}$. Among the foremost priorities to facilitate public interventions is reliable laboratory testing. A valid test is the most effective approach to identify cases in a mass population, including asymptomatic infections, to trace transmission routes and carriers, to evaluate the efficacy of therapeutic approaches, and to determine the eradication of the infection. Therefore, as one of the critical tools in tracing, isolating, and treating COVID-19 pandemics, it is a priority for each country to invest in cutting-edge technologies and to provide financial support for the development and validation of reliable tests for COVID-19. To date, all available tests generally satisfy the demands of mass screening, individual diagnosis or mutation identification, although the capacity varies between countries, regions or races largely because of differences in economic status and healthcare systems. Since the pathogen for COVID-19 is known and the viral genome, transmission routes and host receptor for viral entry are known, currently available tests fall into two categories: (1) nucleic acid-based tests and (2) serology-based tests for detection of viral antigens or host antibodies. Nucleic acid tests directly probe for viral RNA in throat or nasal swabs collected from individuals, whereas serological tests detect antibodies present in serum or viral antigen in tissues, secretions, or eliminations from individuals with ongoing or past infections ${ }^{3}$.

The delineation of the molecular characteristics of the virus helps to develop reliable assays for the detection of viral genomic RNA and proteins. As illustrated in Fig. 1, SARS-CoV-2 is classified as a new $\beta$-coronavirus and possesses a genome composed of positive single-stranded RNA of approximately $30,000 \mathrm{bp}$ nucleotides. SARS-CoV-2 encodes four structural proteins and sixteen nonstructural proteins (NSPs). Structural proteins, including the nucleocapsid $(N)$, envelope glycoprotein spike $(S)$, envelope $(E)$, and transmembrane $(\mathrm{M})$, constitute the envelope and the capsid ${ }^{4}$. The nonstructural proteins encoded by ORF1ab, such as RNAdependent RNA polymerase (RdRp) and helicase (Hel), are mainly required for viral replication ${ }^{5}$. Most molecular diagnoses of COVID19 worldwide involve quantitative reverse-transcription polymerase chain reaction (RT-PCR) assays, and several conserved regions in the SARS-CoV-2 genome have been chosen as reliable targets

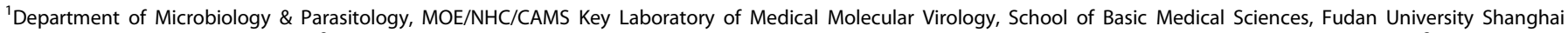

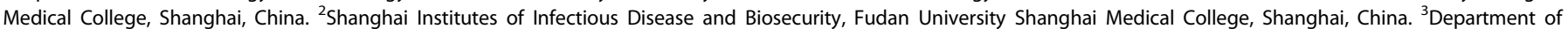

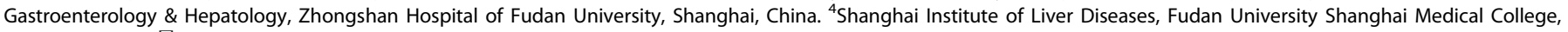
Shanghai, China. ${ }^{凶}$ email: yhxie@fudan.edu.cn; jian.wu@fudan.edu.cn
} 


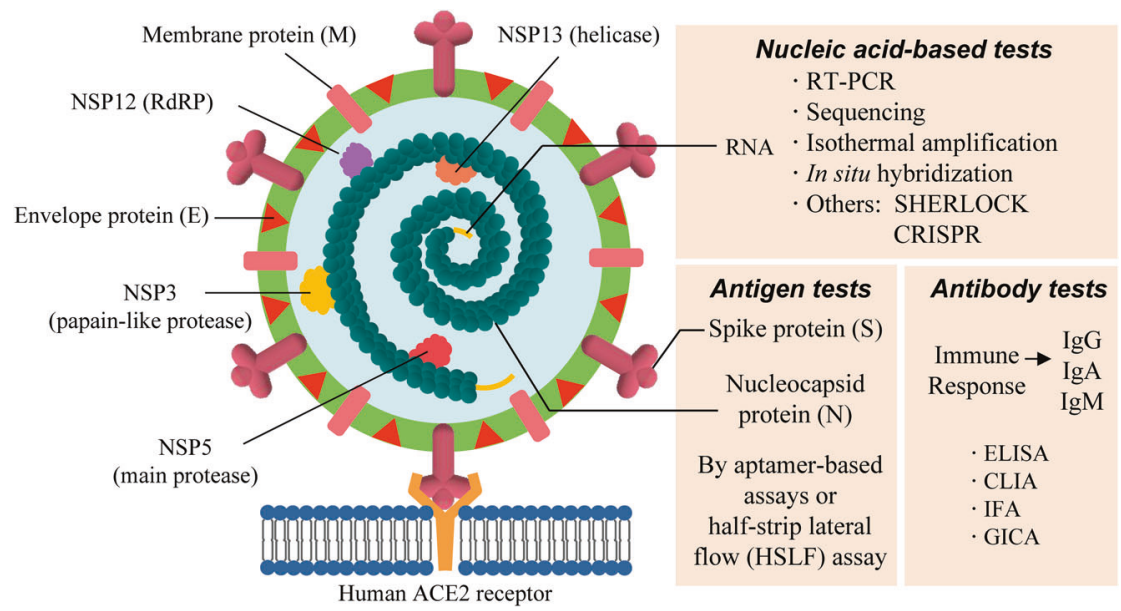

Fig. 1 Illustration of the SARS-CoV-2 viral genome, proteins and corresponding assays. The genome of SARS-CoV-2 is a positive singlestranded RNA with more than 30,000 bp nucleotides. The capsid outside the genome is formed by the nucleocapsid protein ( $\mathrm{N}$ ) and is further wrapped by an envelope composed of three structural proteins: membrane protein (M), spike protein (S), and envelope protein (E). The entry of coronavirus into host cells is mediated by the $S$ protein, which is a homotrimer protruding from the viral envelope that recognizes the receptor angiotensin-converting enzyme 2 (ACE2) via the S1 receptor-binding domain (RBD) and uses the S2 domain for fusion with the host cell membrane to enter host cells. In addition to these four structural proteins, SARS-CoV-2 contains sixteen nonstructural proteins (NSPs). Four NSPs responsible for viral replication or transcription are shown in this illustration. NSP3 separates the translated protein. NSP5 is responsible for cleaving the viral polyprotein into functional units during replication. NSP12 contains the RNA-dependent RNA polymerase (RdRp). NSP13 participates in viral replication or transcription via the zinc-binding domain. ACE2 angiotensin-converting enzyme 2, E envelope protein, $M$ transmembrane protein, $N$ nucleocapsid protein, NSP nonstructural protein, ORF open reading frame, RdRp RNAdependent RNA polymerase, $\mathrm{S}$ spike protein.

for primer design in various PCR assays. In clinical practice, at least two targets are recommended to avoid potential genetic mutation of SARS-CoV-2 or cross-reaction with other coronaviruses, according to the $\mathrm{WHO}^{6}$. Three conserved regions (the $E, N$, and ORF1ab genes) are usually selected as the standard targets for the design of primers and probes (Fig. 2). Moreover, sequencing of the viral genome helps to identify new variants of coronavirus that occur over time. Compared to traditional sequencing methods that are usually very costly, newly emerging portable or quantitative sequencing methods, such as nanopore target sequencing (NTS), may offer accurate high-throughput diagnosis during pandemics.

For serological assays, the $\mathrm{N}$ and $\mathrm{S}$ proteins are the most important targets for immunologic detection among the four structural proteins ${ }^{7}$. For direct detection of viral products, the $\mathrm{N}$ protein, which functions as a structural component of the helical nucleocapsid and plays a vital role in viral replication, is often detected in COVID-19 patients ${ }^{8,9}$. The $S$ protein, which is encoded by the $S$ gene, consists of two subunits, the $S 1$ domain for receptor binding and the S2 domain for fusion, and is critical for receptor recognition, interaction and internalization; therefore, this protein is a particular focus for studies assessing viral mutation and spread $^{4}$.

For most individuals during the first few days of infection, viral titers are high, and a single nasopharyngeal swab may harbor up to 1 million SARS-CoV-2 viral particles. However, patient IgG and IgM antibody production typically occurs 5-10 days after the onset of initial symptoms ${ }^{9}$. Therefore, nucleic acid tests offer the earliest and most sensitive detection for the presence of SARSCoV-2 infection. For research purposes, viral proteins in infected tissues or cells are solid evidence of viral replication, in addition to in situ hybridization for the detection of the viral genome in particular cell types. The titer changes in specific antibodies against viral proteins allow for monitoring the patient response to the infection, and the persistence or fluctuation in antibody levels over time postinfection. As a valid assay, immunologic detection of positivity and titer changes in specific antibodies in a selected population help to determine the mass infection rate, vaccine response, and general immunity against the virus or its variants. Therefore, all these assays have particular usages and may be complementary for prevention, patient care and follow-up, as well as have use in basic and translational research for combating this global threat (Table 1). The present review aims to provide an overview regarding the major advantages, disadvantages, and particular applications of currently available assays for detection, prevention, mass screening, and follow-up in combating this worldwide health crisis.

\section{NUCLEIC ACID TESTING FOR COVID-19}

Quantitative reverse-transcription polymerase chain reaction As an RNA virus, the large genome needs to be reversetranscribed to CDNA for PCR amplification. Hence, quantitative reverse-transcription polymerase chain reaction (qRT-PCR) has been deemed to be the "gold standard" for COVID-19 diagnosis, because it has been shown to be very sensitive for accurately detecting the viral genome, able to detect a single copy of the viral RNA ${ }^{10}$. Three highly conserved regions have been found in the SARS-CoV-2 viral genome, including the $R d R p, E$ and $\mathrm{N}$ gene ${ }^{11}$. The assays are designed as a two-target system in which one primer universally detects numerous coronaviruses, including SARS-CoV-2, and a second primer set exclusively detects SARSCoV-2. For a routine workflow, it is recommended that the $E$ gene be used as the first-line screening target, followed by confirmatory testing of the RdRp gene ${ }^{11}$. A number of RT-PCR primer and probe sets ${ }^{11-14}$ approved for SARS-CoV-2 detection by the Center of Disease Control (CDC) in different countries are listed in Table 2.

The general workflow of RT-PCR tests includes three main steps: sample collection and transport, lysis, and RNA purification and amplification. These standard RT-PCR tests take approximately $3 \mathrm{~h}$ to complete ${ }^{3}$. Efforts have been made to eliminate an RNA purification step, which may dramatically reduce the overall workflow duration in several commercial kits ${ }^{15}$ (Table 3). Notably, the high-throughput TaqPath COVID-19 Combo Kit from Thermo Fisher Scientific detects two copies of the viral genome in one $\mu \mathrm{l}$ of sample, and the detection sensitivity appears to be higher than that of the other kits listed in the table.

A study including 1014 patients found that the average interval between the initial positive and negative RT-PCR results was $6.9 \pm$ 
A

Reference sequence: NC_045512.2

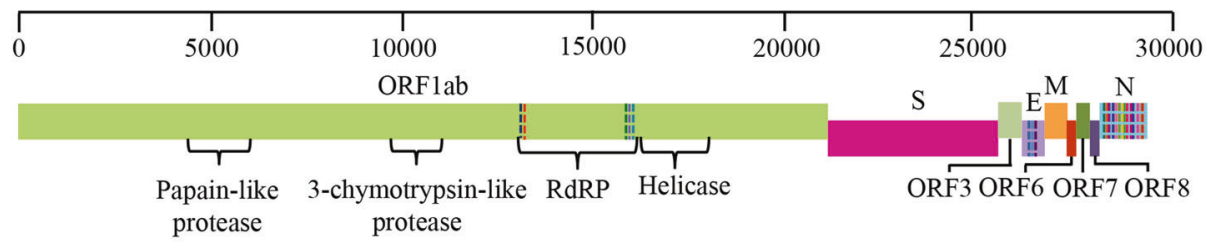

B

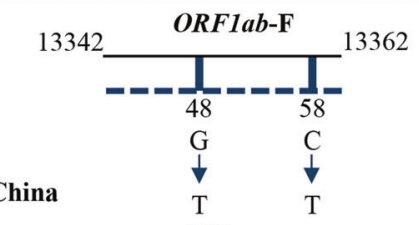

28881 $N-\mathbf{F}$ 28902

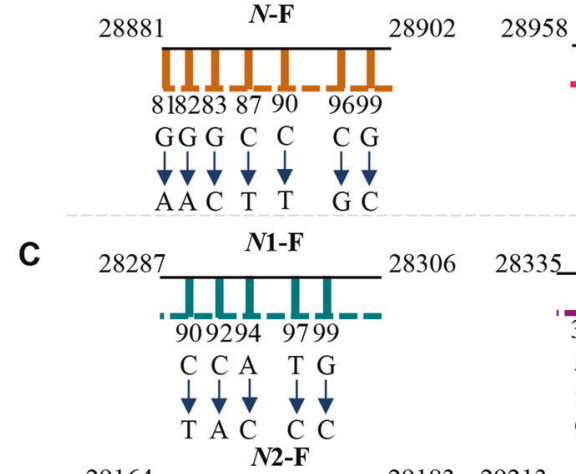

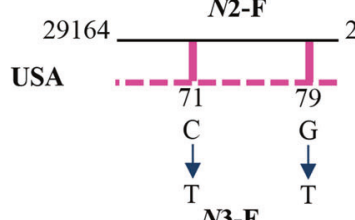
29183

29213

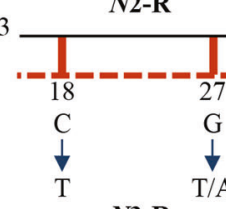

28681

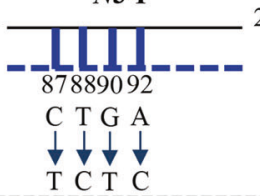
2870228732 N3-R

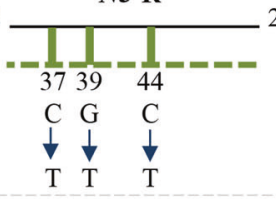

D

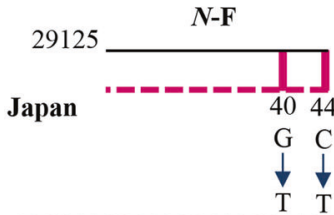
29144


T G T T
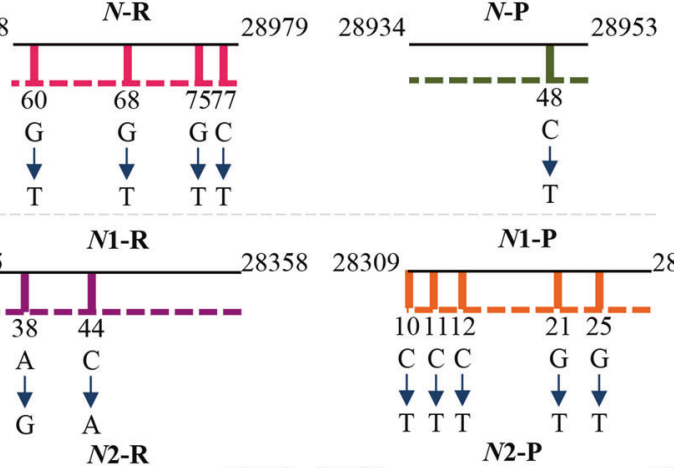
28332

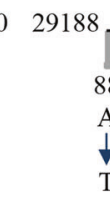

2875228706
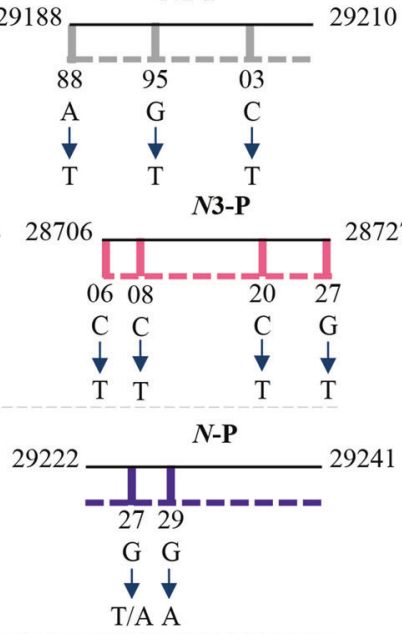
15530

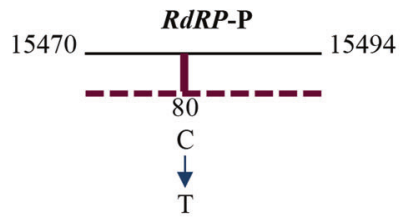

2638126332

E-P
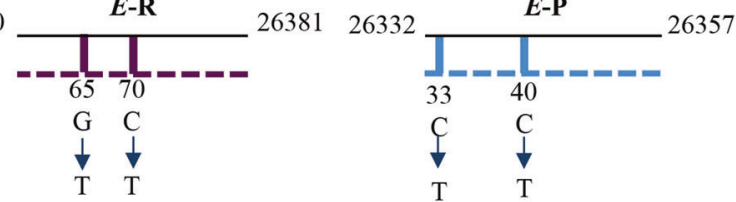

Fig. 2 Viral open reading frames (ORFs) and mutations in primer or probe regions. A Four open reading frames (ORFs) in the viral genome are indicated for encoding viral structural or nonstructural proteins. B-E Mutations have been found in primer and probe regions in various countries and regions. As indicated, most mutations occur in the $\mathrm{N}$ protein region, and the efficiency of primers and probes against the $\mathrm{N}$ protein coding region will be affected by these mutations. Specific point mutations are indicated in primer or probe regions used in various countries, and these mutations may hamper the detection efficiency of RT-PCR kits and cause false-negative results. Therefore, for the detection of newly occurring mutated variants of the SARS-CoV-2 virus, updating specific primers and probes is essential for the reliability of the kits. E envelope protein, $F$ forward primer, $N$ nucleocapsid protein; ORF open reading frame, P probe, R reverse primer, RdRp RNAdependent RNA polymerase. 
Table 1. Common features of various testing methods for SARS-COV-2 infection.

\begin{tabular}{|c|c|c|c|}
\hline Testing type & Time duration (Short or long ${ }^{a}$ ) & Suitable population (Large or small) & Accuracy (High or low) \\
\hline \multicolumn{4}{|l|}{ Nucleic acid testing } \\
\hline Quantitative RT-PCR & Long & Large & High \\
\hline Sequencing for diagnosis & Long & Small & High \\
\hline Isothermal amplification & Short & Large & High \\
\hline In situ hybridization & Long & Small & High \\
\hline \multicolumn{4}{|l|}{ Protein testing } \\
\hline
\end{tabular}

2.3 days ${ }^{16}$. In addition to upper respiratory tract nasopharyngeal swabs, which are mostly used in nucleic acid detection, an observation study detected samples from the digestive tract, including fecal and anal swabs, and found that the clearance time of SARS-CoV-2 in the gastrointestinal tract was longer than that in the respiratory tract ${ }^{17}$, which may last up to 33 days or even longer after a negative respiratory PCR report ${ }^{18}$. Although anal swabs have not been the official standard for diagnosis, they are complementary to the current detection methods to monitor clearance of the virus postinfection. Therefore, it is recommended that inactivation of possible virus-infected human elimination samples in toilets is needed for patient management. It is crucial to choose appropriate sample types and collection routes for monitoring the clearance of SARS-CoV-2 virus. It is equally important to determine how long infected individuals should be isolated depending on serum or fecal sample negativity.

It is worth mentioning that to meet the needs for completion of nucleic acid tests in a large population, the China CDC adopted a pooling method in which samples from at most five different individuals were mixed in one test tube for RT-PCR assays. Once an abnormality is detected in the results of the pooling samples, all individual samples added to the mixed sample are tested separately, which significantly enhances efficiency, as demonstrated by the testing of over 10 million people that was completed within only 19 days in a city in China ${ }^{19}$. It has been recommended that ten samples might be combined together and subjected to nucleic acid extraction and RT-PCR analysis, and the numbers of tests required are estimated based on the incidence of COVID-19 in their respective countries/regions. Compared to $\$ 58$ million for the routine screening of 1 million people, the recommended large-scale population screening method decreases this cost to $\$ 9.1$ million $^{20}$. This cost-effective and time-saving approach has been widely adopted in mass screening tests in large populations during recent local community-spread episodes in several cities in China.

False negativity in RT-PCR tests often occurs, which was observed particularly during the early period of the pandemics, most likely due to improper sample collection, nonstandardized RNA extraction, and an assessment time that was too early for detection of positivity after contact with virus. To avoid false negative results, repeated sample collections are recommended. For successful control of small-scale community spread, up to three nucleic acid tests within 2 weeks have identified potentially contagious "asymptomatic carriers", who are to be quarantined to completely end occasional episodes of local spread in a short period.

In addition to qualitative detection, viral load can also be calculated by plotting CT values onto the standard curve provided by the commercial RT-PCR kits. Differences exist in the viral load of different sample types, as the average viral load in sputum (4 $\log _{10}$ RNA copies/test) is usually higher than that in throat swabs (3 $\log _{10}$ RNA copies/test) and nasal swabs ( $2 \log _{10}$ RNA copies/test) ${ }^{21}$. In respiratory samples, viral load increases with disease severity, and the viral loads of severe patients are at a peak level $\left(6 \log _{10}\right.$ RNA copies $/ \mathrm{mL}$ ) in the third to fourth week after the onset of symptoms, while mild patients reach a peak (5 $\log _{10}$ RNA copies/ $\mathrm{mL}$ ) within one week and slowly decrease $\mathrm{e}^{22}$, which suggested that high viral load might be a risk factor for severe manifestation and a predictor of worse clinical outcome, such as death ${ }^{23}$. On the other hand, the detection of viral load is a critical parameter in evaluating the efficacy of newly developed medications. On February 9, 2021, the U.S. Food and Drug Administration issued an emergency use authorization (EUA) for two monoclonal antibodies (bamlanivimab and etesevimab), which are two anti-spike neutralizing monoclonal antibodies derived separately from two patients that recovered from COVID-19 in North America ${ }^{24}$ and China $^{25}$, to be administered together for the treatment of mild to moderate COVID-19 in adults and pediatric patients ${ }^{26}$. In clinical trials, the primary outcome for characterizing the efficacy of these two neutralizing antibodies was the reduction in SARS-CoV-2 viral load down to baseline, as measured by quantitative RT-PCR ${ }^{27}$.

\section{Detection of mutated variants with standard RT-PCR kits}

The viral genome mutates constantly as it replicates. New variants with genetic mutations may lead to new waves of SARS-CoV-2 pandemic episodes. Since most of the PCR primers have been designed based on the early isolated virions ${ }^{5}$, particularly the reference genome (SARS-CoV-2, NC_045512.2) ${ }^{28}$, even a single mutation in the middle of a primer sequence might contribute to the lower amplification efficiency of GRT-PCR tests and result in false negative results in detection ${ }^{29}$. Studies have analyzed sequencing samples submitted to GenBank and GISAID and found that mutations in Germany and China have mainly occurred in the ORF1ab region ${ }^{30,31}$.

Interestingly, another study based on 31,421 SARS-CoV-2 genome samples found that most of the mutations were within the targets of the various $N$ gene primers and probes ${ }^{32}$, and might affect the efficiency of PCR amplification that is designed to probe the $N$ gene in RT-PCR assays. Cases have been reported that detection might be interfered with due to mutations in the $N$ gene ${ }^{33,34}$. Mutations have been found in all targets of the COVID19 diagnostic primers recommended by the US CDC, whereas the targets of $N$ gene primers and probes used in Japan, Thailand, and China have shown multiple mutations in different clusters, which suggested that the $N$ gene might not be a stable target for RT-PCR kits and that these $N$ gene-based kits should be updated periodically for emerging alpha, beta, gamma, delta variants ${ }^{34}$ (Fig. 2).

\section{Sequencing for diagnosis}

Compared to RT-PCR, viral genome sequencing has the disadvantages of a higher cost, larger amount of data analysis, and 
Table 2. Sequences of RT-PCR primers and probes approved by CDCs in different countries.

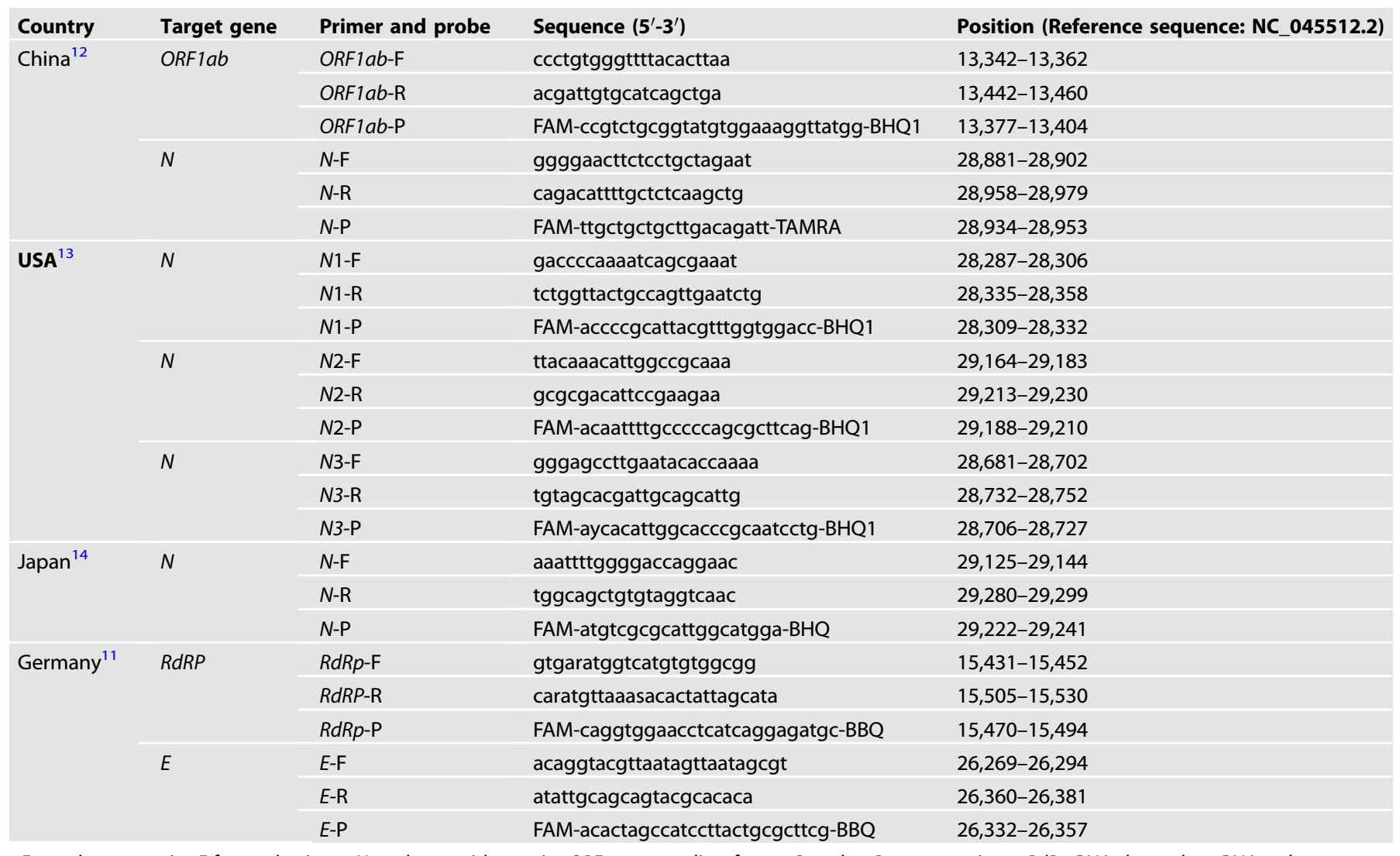

$E$ envelope protein, $F$ forward primer, $N$ nucleocapsid protein, $O R F$ open reading frame, $P$ probe, $R$ reverse primer, $R d R p$ RNA-dependent RNA polymerase.

lower clinical efficiency, which is unsuitable for rapid detection in mass populations. However, the first genome sequence of SARSCoV-2 was precisely achieved using metagenomic RNA sequencing technology ${ }^{28}$. According to the report by the WHO and China, 104 SARS-CoV-2 strains have been isolated and sequenced using Illumina and Nanopore technologies from the end of December 2019 to mid-February $2020^{35}$. The genome and proteome compositions of SARS-CoV-2 have been determined, and over 1000 similar sequences have been made available in the GISAID and GenBank databases ${ }^{14}$. The advantage of sequencing-based detection is that viral mutations can be tracked by collecting information on new strains. Sequencing of the viral genome helps to identify and classify new strains of coronavirus over time ${ }^{35}$. As the virus replicates and spreads, random mutations in the genome accumulate at a rate of approximately two per month, based on the data of closely tracking the viral evolution ${ }^{36}$. New mutant viruses have been reported, including alpha (B.1.1.7), beta (B.1.351), gamma (P.1) and delta (B.1.617.2), which may pose the risk of a much quicker spread of the virus ${ }^{37,38}$.

Due to increasing demand, high-throughput methods or portable rapid sequencing technology have been developed as diagnostic tools for COVID-19. Nanopore target sequencing (NTS) is fast, highly portable, and sensitive, making it attractive for clinical testing. An NTS method sequencing 11 viral regions enable the detection of as few as ten viral copies $/ \mathrm{mL}$ in $1 \mathrm{~h}$ of sequencing ${ }^{39}$. Compared to traditional sequencing methods, which are usually very costly, these newly emerging portable or quantitative methods may provide accurate high-throughput diagnosis during pandemics. A prospective genomic surveillance study in the UK used NTS, enabling sample-to-report in less than $24 \mathrm{~h}$, to establish real-time genomic surveillance of SARS-CoV- ${ }^{40}$.
The combination of genomic and epidemiological analysis accelerates the detection of potential transmission events, and helps to take timely measures to control and prevent widespread of the virus. In addition, a possible pathogenic mechanism might be revealed when NTS is employed to analyze deletions and other mutations in the SARS-CoV-2 genome in infected individuals. Patients infected with virus with deletions mainly in ORF3a and ORF7a of the SARS-CoV-2 genome were observed to be associated with interferon antagonism ${ }^{41}$. Moreover, a novel molecular diagnostic tool based on Sanger sequencing technology was able to detect SARS-CoV-2 RNA from viral particles suspended in transmission medium (directly added to the PCR master mix), suggesting that RNA extraction may be skipped completely without reducing performance at a testing speed of more than $1,000,000$ tests per day ${ }^{42}$. With this capacity, one may imagine that natural mutations in mass populations can be mapped at overall genome levels or specific sites during a particular time period or within a geographic area, allowing the sources and origins of the variants to be traced when analytic capability is in place.

\section{Isothermal amplification}

RT-PCR is performed in a thermal cycle device, which is under precise temperature control and needs a power supply. In contrast, isothermal amplification technology is carried out at a constant temperature using a specific enzyme for rapid nucleic acid amplification. The reaction takes place generally at $60-65^{\circ} \mathrm{C}$ and is completed within $1 \mathrm{~h}^{43}$, conferring an analytic sensitivity similar to PCR without special laboratory equipment such as a thermal cycler ${ }^{44}$. The isothermal technique utilizes a recombinant polymerase and helicase-dependent or loop-mediated isothermal 
Table 3. Features of commercial kits for COVID-19 nucleic acid detection.

\begin{tabular}{|c|c|c|c|c|c|}
\hline Kit name & Manufacturer & Target genes & 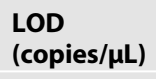 & Accuracy (\%) & TAT (min) \\
\hline TRUPCR SARS-CoV-2 RT-qPCR ${ }^{15}$ & Black Biotech & $E, N \& R d R p$ & 10 & 100.0 & Unavailable \\
\hline Allplex 2019-nCoV assay ${ }^{15}$ & Seegene & $E, N \& R d R p$ & 4167 & 100.0 & 110 \\
\hline LabGun real-time $P C R^{15}$ & Lab Genomics & $E \& R d R p$ & 20 & 94.3 & Unavailable \\
\hline
\end{tabular}

$E$ envelope protein, $L O D$ limit of detection, $M$ transmembrane protein, $N$ nucleocapsid protein, ORF open reading frame, $R d R p$ RNA-dependent RNA polymerase, $S$ spike protein, TAT turn-around time.

Table 4. SARS-CoV-2 RT-LAMP tests in different laboratories.

\begin{tabular}{|c|c|c|c|c|}
\hline Authors & Target genes & LOD (copy/reaction) & Sensitivity and specificity & TAT (min) \\
\hline Lu et al. $^{46}$ & $N, S$ and $R d R p$ & 118.6 & 94 and $90 \%$ & 20 \\
\hline Huang et al. ${ }^{47}$ & $N, S$ and $R d R p$ & 20 & 100 and $100 \%$ & 30 \\
\hline Yan et al. ${ }^{48}$ & ORF1a/b & 20 & 100 and $100 \%$ & 60 \\
\hline Baek et al. ${ }^{49}$ & $N$ & 100 & 100 and $98.70 \%$ & 30 \\
\hline Lu et al. $^{50}$ & $R d R p$ & 30 & $100 \% /-$ & 40 \\
\hline Jiang et al. ${ }^{51}$ & ORF $1 b$ and $N$ & 500 copies $/ \mathrm{mL}$ & 91.4 and $99.5 \%$ & 30 \\
\hline Kitawaya et al. ${ }^{52}$ & $N$ and $S$ & 10 copies $/ \mu \mathrm{L}$ & 100 and $97.6 \%$ & 35 \\
\hline
\end{tabular}

LOD limit of detection, $N$ nucleocapsid protein, ORF open reading frame, RdRp RNA-dependent RNA polymerase, $S$ spike protein, TAT turn-around time.

amplification (LAMP) and allows fast, sensitive, portable, and point-of-care applications.

Reverse transcription LAMP (RT-LAMP) has been validated for the detection of several RNA viruses, including influenza, Zika, Ebola, and MERS. The positive reaction of LAMP is quantified visually by turbidity, colorimetric and fluorometric parameters, because the insoluble byproduct magnesium pyrophosphate formed during the LAMP reaction is visible to the naked eye ${ }^{45}$. RT-LAMP tests for SARS-CoV-2 have already been developed and clinically validated worldwide with the shortest reaction time of $20 \mathrm{~min}$ and the lowest detection limit of 20 copies of the virion

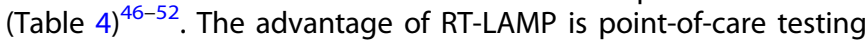
without the requirement for specific equipment, which is particularly valuable in a remote region or a large population. For the demands of a high-throughput capacity, automation for sample handling, assay performance and result reports are the keys for wide acceptance in pandemic epicenters.

\section{Other nucleic acid-based assays}

SHERLOCK (specific high sensitivity enzymatic reporter unlocking) is a system using Cas13a ribonuclease for RNA detection ${ }^{53}$. The SHERLOCK procedure consists of two main steps: isothermal target nucleic acid amplification and CRISPR-Cas13 nucleic acid detection. Cas 13 is an RNA-guided RNase that produces multiple cleavage sites in single-stranded areas of an RNA target with a specific base preference and has been shown to be useful for nucleic acid detection in CRISPR-based systems ${ }^{54}$. CRISPR-Cas 13 enzymes are programmed by CRISPR RNA (crRNA) and exhibit nonspecific endonuclease activity after binding to a specific target. As illustrated in Fig. 3, once the CRISPR-Cas13-RNA complex is activated by binding to a complementary RNA target through recombinase polymerase amplification, the fluorescent quencher probe around the cleavage site produces fluorescent signaling ${ }^{55}$. This detection system has been applied for the nucleic acid detection of Zika virus ${ }^{56}$ and the point-of-care testing for COVID-
19 in a maximal capacity of 100 samples per hour ${ }^{57}$. On the basis of the two-step process, a streamlined assay that combines the simplified extraction of viral RNA with isothermal amplification and CRISPR-mediated detection, designated as SHERLOCK Testing in One Pot (STOP), has been developed ${ }^{54}$. A common reaction buffer that accommodates both steps has been developed, and the test is performed at a single temperature in less than an hour with a low risk of cross-contamination.

Other CRISPR-based detection assays are also currently in use. One method that utilizes a custom CRISPR-Cas12a/gRNA complex, and a fluorescent probe to detect target amplicons produced by standard RT-PCR allows sensitive and robust detection of SARSCoV-2-positive samples, with a sample-to-answer time within 50 min and a limit of detection (LOD) of two copies per reaction ${ }^{58}$. The AIOD-CRISPR system uses a pair of Cas12a-crRNA complexes, which may bind to corresponding sites close to the primer recognition sites in the target sequence, and is visually detected by fluorescence or color change ${ }^{59}$. A plasmid containing a cDNA representing 384 nucleotides of the $N$ gene has already been used as the target to develop an all-in-one dual CRISPR-Cas12a (AIODCRISPR) assay, which was shown to detect 1.3 copies of the SARSCoV-2 $N$ gene plasmid in a visual and real-time mode within 40 $\min ^{60}$. Another assay, known as DNA endonuclease-targeted CRISPR trans-reporter (DETECTR), has been designed to simultaneously reverse transcribe and isothermally amplify the RNA extracted from nasopharyngeal swabs, followed by Cas12mediated viral detection ${ }^{61}$. The DETECTR assay provides a visual and rapid $(<40 \mathrm{~min})$ alternative with a $95 \%$ positive prediction value.

The above-mentioned CRISPR-based assays are rapid and visual, and may avoid complex laboratory infrastructure. They are also highly sensitive and specific with a low LOD (1-2 copies). SHERLOCK may distinguish Zika variants from Honduras, the Dominican Republic, and the US, even in sequence regions that differ by only a single nucleotide ${ }^{62}$. Despite these advantages, 


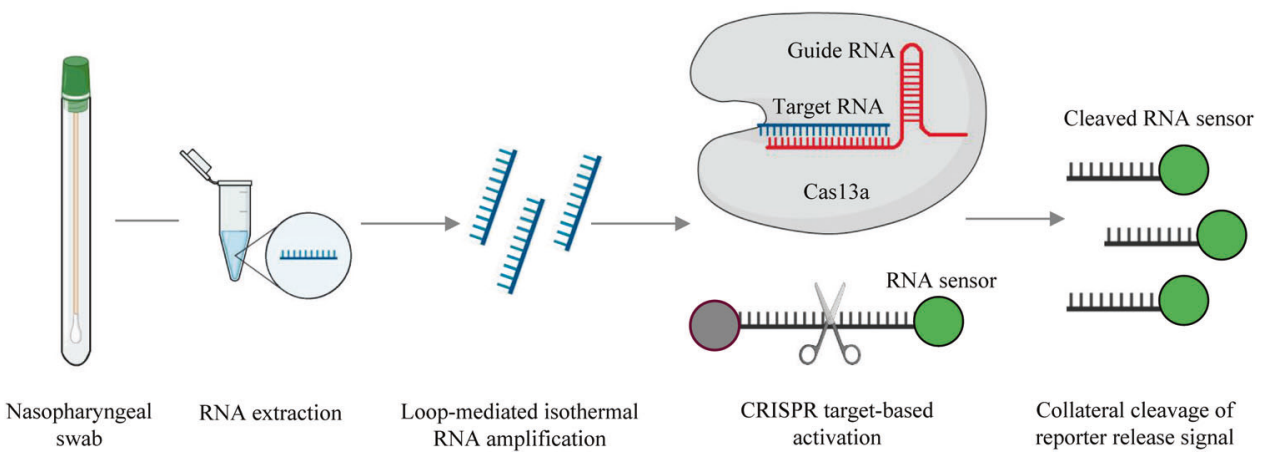

Fig. 3 A schematic illustration of the SHERLOCK detection assay. Using nasopharyngeal swabs as an example, conventional RNA extraction is used as the input and is followed by reverse transcription and loop-mediated isothermal amplification. The CRISPR-Cas13-RNA complex is activated by binding to a complementary RNA target, while CRISPR-Cas13 exhibits nonspecific endonuclease activity, which activates and cleaves fluorescent RNA sensors. The fluorescent RNA sensor is quenched when it is intact, whereas it emits fluorescent signals when it is cleaved by the activated CRISPR-Cas13 complex.

they have several limitations for clinical validation. Since no commercial kit is available, the existing CRISPR-based assays rely on the preparation and testing of reaction components, such as bacterial transformation and large-scale protein expression. These empirical adjustments of parameters require expertize in protein purification and RNA biology. Compared to RT-PCR, the multistep nucleic acid amplification process may affect precise target quantification in CRISPR-based assays. Raw nucleic acid extractions may be contaminated with nucleases, which may degrade viral samples and generate false-negative signals; at the same time, they may degrade the sensing molecules and lead to false positive signals. When designing crRNA, overlapping the recombinase and the polymerase coding regions should be avoided so as not to detect off-target products. Because of the preamplification reaction, the risk of surface contamination might increase in the multistep assays. Thus, for applications that do not demand speed but do demand quantitative detection, a clinically validated detection assay, such as RT-PCR, might be a better choice. A comparison of the CRISPR-based assays with the qRT-PCR assay is listed in Table 5.

In situ hybridization for viral RNA detection in tissues or cells To determine which tissue or cell types are susceptible to SARSCoV-2 infection, the presence of the ACE2 receptor is the key for selective viral entry. ACE2 is widely present in the upper respiratory tract and lungs; thus, the lungs are typically highly infected and are the main site for viral replication. To determine whether other tissues, such as the heart, liver, and kidneys, are also targets of infection, in addition to detection of the ACE2 receptor and viral proteins in these tissues by immunohistochemical staining, detection of viral genomic RNA by in situ hybridization is a classic and reliable approach necessary to pinpoint viral replication in the cell types of the infected tissue. For example, viral RNA positivity was observed in the placenta in a SARS-CoV-2positive pregnant woman, and maternal-fetal transmission was confirmed after infection ${ }^{63}$. Thus, in situ hybridization as a molecular approach may be used as a research tool in verifying viral replication in particular cell types or tissues, and yields additional histological evidence of viral infection after antibody detection of viral protein by immunohistochemical staining. More solid and direct evidence of infection would be electronic microscopy observation of viral particles in the tissue or cells ${ }^{64-66}$.

In summary, among all the nucleic acid detection methods of SARS-CoV-2, qRT-PCR is more commonly used due to its higher sensitivity and higher specificity, which enables rapid screening of a large number of specimens within a short time. As summarized in Tables 2-4, several commercial RT-PCR kits have been developed and widely used. Special attention should be paid
Table 5. Comparison of CRISPR-based and RT-PCR assays.

\begin{tabular}{|c|c|c|}
\hline $\begin{array}{l}\text { Parameters or } \\
\text { aspects }\end{array}$ & $\begin{array}{l}\text { CRISPR- } \\
\text { based assays }\end{array}$ & RT-PCR assays \\
\hline Target gene & $E$ and $N$ & $\begin{array}{l}\text { ORF1ab, N, E, and } \\
\text { RdRP }\end{array}$ \\
\hline TAT & $30-40 \mathrm{~min}$ & $4 \mathrm{~h}$ \\
\hline Assay results & Qualitative & Quantitative \\
\hline $\begin{array}{l}\text { Specific instrument } \\
\text { required }\end{array}$ & No & Yes \\
\hline FDA or EUA approval & Not yet & Yes \\
\hline
\end{tabular}

$E$ envelope protein, $N$ nucleocapsid protein, EUA European emergency use authorization, FDA US Food and Drug Administration, ORF open reading frame, RdRp RNA-dependent RNA polymerase, TAT turn-around time.

particularly to the occurrence of false negative results when using primers and probes for the $N$ gene target, given that previous studies have confirmed that mutations in the $N$ gene might lead to interference in the test. Viral genome sequencing takes a longer time at a higher expense than qRT-PCR assays and makes it unsuitable for diagnosis and patient screening. Instead, the availability of whole viral sequence information from different regions allows for tracing the source and mutations of SARS-CoV-2 in a specific region or population. The main challenge for using sequencing as a tool for combating the pandemic is how to reduce the detection duration and cost. RT-LAMP usually allows detection within $1 \mathrm{~h}$ under isothermal conditions without the need for special equipment. Moreover, the results are visible to the naked eye, which is suitable for viral detection in remote areas or other places lacking thermal cyclers. In addition, new molecular detection methods, such as CRISPR-based assays, focus on how to further reduce time and cost while maintaining high sensitivity and specificity. For research purposes, traditional in situ hybridization validates the presence of the viral genome in particular tissue or cell types and provides direct evidence of viral replication in tissue or cell types. The selection of particular methods or modes of detection depends largely on the population size and the purpose, such as for diagnosis, tracing, or laboratory research.

\section{PROTEIN-BASED COVID-19 DIAGNOSIS Antibody testing}

As an increasing number of people worldwide have insisted on maintaining social distance and staying at home, the focus of epidemic prevention and control has now shifted to extensive serological antibody testing of the population to monitor 
Table 6. Features of different antibody tests.

\begin{tabular}{|c|c|c|c|c|}
\hline Kit name & Manufacturer & Test type & Antibody tested & Sensitivity and specificity \\
\hline Platelia SARS-CoV-2 total antibody assay ${ }^{84}$ & Bio-Rad & ELISA & Total antibody & 100.0 and $99.6 \%$ \\
\hline COVID-19 antibody test ${ }^{84}$ & Mount Sinai & ELISA & $\lg G$ & 92.0 and $100.0 \%$ \\
\hline LIAISON ${ }^{\circledR}$ SARS-CoV-2 S1/S2 lgG ${ }^{84}$ & DiaSorin & CLIA & $\lg G$ & 97.56 and $99.3 \%$ \\
\hline Eugene $^{\circledR}$ SARS-CoV2 lgG/lgM rapid test ${ }^{85}$ & Shanghai Eugene Biotech & GICA & Combo IgM/lgG & 96.4 and $98.7 \%$ \\
\hline Standard ${ }^{\mathrm{TM}} \mathrm{Q}$ COVID-19 $\mathrm{lgM} / \operatorname{lgG}$ duo test ${ }^{85}$ & SD Biosensor & GICA & Combo lgM/lgG & $100 \%$ and $\lg M \quad 91.7 \%$, IgG $79.2 \%$ \\
\hline
\end{tabular}

CLIA chemiluminescent immunoassay, ELISA enzyme-linked immunosorbent assay, GICA colloidal gold immunochromatographic assay, IFA immunofluorescent assay, IgG immunoglobulin $G$, IgM immunoglobulin $M$.

population infection status, vaccine efficacy, immunity persistence, and high-titer neutralizing antibody screening and collection. These tests, such as enzyme-linked immunosorbent assay (ELISA), chemiluminescent immunoassay (CLIA), immunofluorescent assay (IFA) and colloidal gold immune chromatographic assay (GICA), are based on targeting $S$ protein and $\mathrm{N}$ protein antigens for the rapid detection of SARS-CoV-2 through $\operatorname{lgM}$ and/or $\lg G$ antibodies in serum or body fluid samples (Table 6). One study investigated the presence of SARS-CoV-2-specific antibodies in 959 blood samples collected from a prospective pulmonary cancer screening among asymptomatic individuals in Italy between September 2019 and March 2020, several months before the first patient was reported ${ }^{67}$. Testing indicates that SARS-CoV-2 infection was present in approximately $11.6 \%$ of the local population before COVID-19 was initially reported.

The usefulness of antibody tests is for population exposure studies to investigate the exposure rate before and after a major epidemic episode in a region, and to determine whether neutralizing antibodies are developing in individuals, who have been exposed to the virus and the duration and titer changes in neutralizing antibodies over time. It is critical to follow-up on neutralizing antibody development after vaccination since various types of vaccines against SARS-CoV-2 infection are available for general populations.

\section{Antigen testing}

In SARS-CoV, $\mathrm{N}$ protein and $\mathrm{S}$ protein are the main immunogens, and antibodies against these two proteins may last for 30 weeks in the serum of SARS patients ${ }^{68}$. A novel antigen-based rapid test for diagnosis showed high sensitivity and specificity mainly in the first week among symptomatic patients and samples with high viral load $^{69}$, while a rapid method based on a fluorescent immune chromatographic assay detecting $\mathrm{N}$ protein demonstrated high sensitivity only in an early phase of infection ${ }^{70}$. Mass spectrometry analysis reported the presence of $\mathrm{N}$ protein in gargle solution samples of COVID-19 patients ${ }^{71}$. A fluorescent immune chromatographic assay detected $\mathrm{N}$ protein in urine samples in $73.6 \%$ of diagnosed COVID-19 patients ${ }^{70}$. Due to its late appearance, $\mathrm{S}$ protein is more suitable for detection during the recovery period $^{72}$, and an ultrasensitive antigen test for $S$ protein is conveniently performed with a microplate reader ${ }^{73}$.

In addition to these common methods, the SARS-CoV-2 coronavirus nucleocapsid antigen-detecting half-strip lateral flow (HSLF) assay has been developed, which displays better clinical sensitivity than traditional serology assays, as the LOD for the commercially available Genscript $N$ protein is $3.03 \mathrm{ng} / \mathrm{mL}^{74}$. A novel nanozyme-based chemiluminescent paper assay is feasible using the camera of a standard smartphone, with a LOD for recombinant spike antigen of SARS-CoV- 2 of $0.1 \mathrm{ng} / \mathrm{mL}^{75}$.

For antigen detection, a specific nucleotide aptamer against the $\mathrm{N}$ protein has the same specificity as an antibody for recognizing the target; however, it may have better sensitivity and more options for the development of assays for different purposes. Through a SELEX selection strategy ${ }^{76}$, a specific ssDNA aptamer that binds to the $\mathrm{N}$ protein has been suggested to be a sensitive and alternative probe for the detection of SARS-CoV- $2^{77}$. Moreover, another study reported that four DNA aptamers with an affinity below $5 \mathrm{nM}$ were identified to bind to the $\mathrm{N}$ protein in a sandwich-type interaction with an LOD of $1 \mathrm{ng} / \mathrm{mL}^{78}$. Compared to using antibodies alone in ELISA with LODs ranging from 50 to 100 $\mathrm{ng} / \mathrm{mL}^{79,80}$, the LOD of aptamer-based technologies was much lower than that of common immunoassays in a short turn-around time (TAT) with high repeatability and reusability ${ }^{76,81}$. Therefore, aptamer-based antigen detection may be superior to antibodybased assays in terms of detection sensitivity, versatility in biosensor conjugation for chemiluminescent or fluorescent detection, and much lower variation in aptamer production ${ }^{76,81}$.

Finally, it is worth mentioning that the sensitivity of rapid antigen detection is $10^{3}$ times lower than that of virus culture and $10^{5}$ times lower than that of RT-PCR ${ }^{82}$. Previous studies reported that the sensitivity of the rapid antigen test is approximately only $30 \%$ of nucleotide acid testing ${ }^{83}$, suggesting that antigen testing is not a rapid method but may be used as a confirmation or research assay for specific patient samples.

\section{PERSPECTIVES AND CONCLUSION}

The global COVID-19 pandemic is one of the most devastating infectious diseases in history in terms of infection numbers and mortality in humans, and heavily hit areas are still in combating the overwhelming hospitalization rate and fatality, although vaccination is ongoing for high-risk populations. The emergence and epidemic of new variants in more than 20 countries have led to a high surge and more rapid transmission in affected regions. Mutated variants may bring about new challenges in false negativity in currently available diagnostic nucleic acid detection and in the efficacy of currently available mRNA-based, recombinant, or inactivated vaccines. Recently, re-emerging communityacquired transmission in China due to the foreign travel of people or the import of goods has led to the implementation of largescale population screening and tracing, which has resulted in the timely control of community-acquired and small-scale epidemics. Therefore, identifying infectious sources, such as asymptomatic individuals, infected individuals or contaminated goods, has become an effective measure in containing community spread.

As discussed, among the nucleic acid-based methods, quantitative RT-PCR is the most specific and the fastest method for screening and diagnosis in a large population, and the sequencing of the viral genome is the most reliable method in tracing infectious sources, monitoring mutations, and determining genome types with limited capacity for selected individuals. Viral load determination by quantitative RT-PCR is valid for monitoring 
disease progression, therapeutic efficacy, and prognosis. Emerging new technologies, such as RT-LAMP and CRISPR-based assays, offer fast point-of-care testing for heavily infected or remote areas. For research purposes, in situ RNA hybridization for direct detection of the viral RNA genome yields convincing evidence of infection in specific tissue or cell types. Using specific antibodies to detect viral proteins, such as the $\mathrm{N}$ or $\mathrm{S}$ proteins, in tissue by immunohistochemical assays allows the quick observation of virus-infected tissue distribution in particular organs, tissues or cell types. For viral protein detection, the halfstrip lateral flow (HSLF) assay is a state-of-the-art advancement in providing convenient point-of-care detection in remote regions or self-service at home. Moreover, aptamer-based assays may have the same level of specificity as antibody-based assays, but possess a lower LOD with more options for high-throughput capacity. For antibody testing, although its sensitivity is lower than that of quantitative RT-PCR, various methods, such as ELISA and fluorescent or luminescent assays, have been developed to detect IgG or IgM postinfection. These assays have wide applications in tracing infected individuals in a large population and monitoring specific antibody development and persistence. Moreover, they are useful for selecting individuals with high titers of neutralizing antibodies for therapy or prevention as a passive immunity approach. It is known that antibody assays are a primary method to determine immunity development and effectiveness, as well as persistence after vaccination. Newly emerging assays, such as RTLAMP, SHERLOCK, AIOD-CRISPR, and DETECTR, are required to combat pandemics and evolve for specific applications. To select the right assay for a particular usage, understanding its principles, the advantages and disadvantages is essential to fulfill this task. With the availability of multiple options for the detection of viral nucleic acid and protein or host antibody production, specific and effective assays aid in identifying infectious sources, assigning isolation or diagnosis, and meeting research demands in combating this global pandemics, which is expected to be under control due to widespread vaccination in general populations in the coming months or years.

\section{DATA AVAILABILITY}

No original data are presented in this review article. Summaries of published data are presented in Tables 1-6.

\section{REFERENCES}

1. Zhou, P. et al. A pneumonia outbreak associated with a new coronavirus of probable bat origin. Nature 579, 270-273 (2020).

2. Coronaviridae Study Group of the International Committee on Taxonomy of 500 Viruses. The species Severe acute respiratory syndrome-related coronavirus: classifying 2019-nCoV and naming it SARS-CoV-2. Nat. Microbiol. 5, 536-544 (2020).

3. Esbin, M. N. et al. Overcoming the bottleneck to widespread testing: a rapid review of nucleic acid testing approaches for COVID-19 detection. RNA 26, 771-783 (2020)

4. Cui, J., Li, F. \& Shi, Z. L. Origin and evolution of pathogenic coronaviruses. Nat. Rev. Microbiol. 17, 181-192 (2019).

5. Chan, J. F. et al. Improved molecular diagnosis of COVID-19 by the novel, highly sensitive and specific COVID-19-RdRp/Hel real-time reverse transcription-PCR assay validated in vitro and with clinical specimens. J. Clin. Microbiol. 58, e00310-e00320 (2020).

6. Holshue, M. L. et al. First case of 2019 novel coronavirus in the United States. N. Engl. J. Med. 382, 929-936 (2020).

7. Tang, Y. W., Schmitz, J. E., Persing, D. H. \& Stratton, C. W. Laboratory diagnosis of COVID-19: current issues and challenges. J. Clin. Microbiol. 58, e00512-e00520 (2020).

8. Chan-Yeung, M. \& Xu, R. H. SARS: epidemiology. Respirology 8, S9-14 (2003).

9. Liu, Y., Eggo, R. M. \& Kucharski, A. J. Secondary attack rate and superspreading events for SARS-CoV-2. Lancet 395, e47 (2020).

10. Wang, J. et al. Novel one-step single-tube nested quantitative real-time PCR assay for highly sensitive detection of SARS-CoV-2. Anal. Chem. 92, 9399-9404 (2020).
11. Corman, V. M. et al. Detection of 2019 novel coronavirus (2019-nCoV) by real-time RT-PCR. Euro Surveill. 25, 2000045 (2020).

12. China CDC. The detection primer and probe sequences of SARS-CoV-2. http:// ivdc.chinacdc.cn/kyjz/202001/t20200121_211337.html (2020).

13. US CDC. Information about emergency use authorization for 2019 novel coronavirus (2019-nCoV) real-time RT-PCR diagnostic panel. https://www.cdc.gov/ csels/dls/locs/2020/information_about_emergency_use_authorization_for_ 2019_novel_coronavirus_real_time_rt-pcr_diagnostic_panel.html (2020).

14. Udugama, B. et al. Diagnosing COVID-19: the disease and tools for detection. ACS Nano 14, 3822-3835 (2020).

15. Garg, A. et al. Evaluation of seven commercial RT-PCR kits for COVID-19 testing in pooled clinical specimens. J. Med. Virol. 93, 2281-2286 (2020).

16. $\mathrm{Ai}, \mathrm{T}$. et al. Correlation of chest $\mathrm{CT}$ and RT-PCR testing for coronavirus disease 2019 (COVID-19) in China: a report of 1014 cases. Radiology 296, E32-E40 (2020).

17. Wu, J. et al. Detection and analysis of nucleic acid in various biological samples of COVID-19 patients. Travel Med. Infect. Dis. 37, 101673 (2020).

18. Cheung, K. S. et al. Gastrointestinal manifestations of SARS-CoV-2 infection and virus load in fecal samples from a Hong Kong cohort: systematic review and meta-analysis. Gastroenterology 159, 81-95 (2020).

19. The State Council Information Office of the People's Republic of China. The 104th press conference on the prevention and control of COVID-19 in Hubei province. http://www.scio.gov.cn/xwfbh/gssxwfbh/xwfbh/hubei/document/1681627/ 1681627.htm (2020).

20. Sahajpal, N. S. et al. Proposal of RT-PCR-based mass population screening for severe acute respiratory syndrome Coronavirus 2 (Coronavirus disease 2019). J. Mol. Diagn. 22, 1294-1299 (2020).

21. $\mathrm{Yu}, \mathrm{F}$. et al. Quantitative detection and viral load analysis of SARS-CoV-2 in infected patients. Clin. Infect. Dis. 71, 793-798 (2020).

22. Zheng, S. et al. Viral load dynamics and disease severity in patients infected with SARS-CoV-2 in Zhejiang province, China, January-March 2020: retrospective cohort study. BMJ 369, m1443 (2020).

23. Chu, C. M. et al. Initial viral load and the outcomes of SARS. CMAJ 171, 1349-1352 (2004).

24. FDA. Coronavirus (COVID-19) Update: FDA authorizes monoclonal antibodies for treatment of COVID-19, https://www.fda.gov/news-events/press-announcements/ coronavirus-covid-19-update-fda-authorizes-monoclonal-antibodies-treatmentcovid-19-0 (2021).

25. Jones, B. E. et al. LY-CoV555, a rapidly isolated potent neutralizing antibody, provides protection in a non-human primate model of SARS-CoV-2 infection. bioRxiv, Preprint posted online 9 October 2020, https://doi.org/10.1101/ 2020.09.30.318972 (2020).

26. Shi, R. et al. A human neutralizing antibody targets the receptor-binding site of SARS-CoV-2. Nature 584, 120-124 (2020).

27. Gottlieb, R. L. et al. Effect of bamlanivimab as monotherapy or in combination with etesevimab on viral load in patients with mild to moderate COVID-19: a randomized clinical trial. JAMA 325, 632-644 (2021).

28. Wu, F. et al. A new coronavirus associated with human respiratory disease in China. Nature 579, 265-269 (2020)

29. Bru, D., Martin-Laurent, F. \& Philippot, L. Quantification of the detrimental effect of a single primer-template mismatch by real-time PCR using the 16S rRNA gene as an example. Appl. Environ. Microbiol. 74, 1660-1663 (2008).

30. Farkas, C., Fuentes-Villalobos, F., Garrido, J. L., Haigh, J. \& Barría, M. I. Insights on early mutational events in SARS-CoV-2 virus reveal founder effects across geographical regions. Peer」 8, e9255 (2020).

31. Koyama, T., Platt, D. \& Parida, L. Variant analysis of SARS-CoV-2 genomes. Bull. World Health Organ. 98, 495-504 (2020).

32. Wang, R., Hozumi, Y., Yin, C. \& Wei, G. W. Mutations on COVID-19 diagnostic targets. Genomics 112, 5204-5213 (2020).

33. Ziegler, K. et al. SARS-CoV-2 samples may escape detection because of a single point mutation in the N gene. Euro Surveill. 25, 2001650 (2020).

34. Kaden, R. Early phylogenetic diversification of SARS-CoV-2: determination of variants and the effect on epidemiology, immunology, and diagnostics. J. Clin. Med. 9, 1615 (2020).

35. Lu, R. et al. Genomic characterisation and epidemiology of 2019 novel coronavirus: implications for virus origins and receptor binding. Lancet 395, 565-574 (2020).

36. Hadfield, J. et al. Nextstrain: real-time tracking of pathogen evolution. Bioinformatics 34, 4121-4123 (2018)

37. Tang, J. W., Tambyah, P. A. \& Hui, D. S. Emergence of a new SARS-CoV-2 variant in the UK. J. Infect. 82, e27-e28 (2020).

38. Makoni, M. South Africa responds to new SARS-CoV-2 variant. Lancet 397, 267 (2021). 
39. Wang, M. et al. Nanopore target sequencing for accurate and comprehensive detection of SARS-CoV-2 and other respiratory viruses. Small 16, e2002169 (2020).

40. Meredith, L. W. et al. Rapid implementation of SARS-CoV-2 sequencing to investigate cases of health-care associated COVID-19: a prospective genomic surveillance study. Lancet Infect. Dis. 20, 1263-1272 (2020).

41. Moore, S. C. et al. Amplicon-based detection and sequencing of SARS-CoV-2 in nasopharyngeal swabs from patients with COVID-19 and identification of deletions in the viral genome that encode proteins involved in interferon antagonism. Viruses 12, 1164 (2020)

42. Chandler-Brown, D., Bueno, A. M., Atay, O. \& Tsao, D. S. A highly scalable and rapidly deployable RNA extraction-free COVID-19 assay by quantitative Sanger sequencing. bioRxiv https://doi.org/10.1101/2020.04.07.029199 (2020).

43. Notomi, T. et al. Loop-mediated isothermal amplification of DNA. Nucleic Acids Res. 28, E63 (2000)

44. Craw, P. \& Balachandran, W. Isothermal nucleic acid amplification technologies for point-of-care diagnostics: a critical review. Lab Chip 12, 2469-86 (2012).

45. Mori, Y. \& Notomi, T. Loop-mediated isothermal amplification (LAMP): a rapid, accurate, and cost-effective diagnostic method for infectious diseases. J. Infect Chemother. 15, 62-69 (2009).

46. Lu, R. et al. A novel reverse transcription loop-mediated isothermal amplification method for rapid detection of SARS-CoV-2. Int. J. Mol. Sci. 21, 2826 (2020).

47. Huang, W. E. et al. RT-LAMP for rapid diagnosis of coronavirus SARS-CoV-2. Microb. Biotechnol. 13, 950-961 (2020).

48. Yan, C. et al. Rapid and visual detection of 2019 novel coronavirus (SARS-CoV-2) by a reverse transcription loop-mediated isothermal amplification assay. Clin. Microbiol. Infect. 26, 773-779 (2020).

49. Baek, Y. H. et al. Development of a reverse transcription-loop-mediated isothermal amplification as a rapid early-detection method for novel SARS-CoV-2. Emerg. Microbes Infect. 9, 998-1007 (2020).

50. Lu, R. et al. Development of a novel reverse transcription loop-mediated isothermal amplification method for rapid detection of SARS-CoV-2. Virol. Sin. 35, 344-347 (2020).

51. Jiang, M. et al. Development and validation of a rapid, single-step reverse transcriptase loop-mediated isothermal amplification (RT-LAMP) system potentially to be used for reliable and high-throughput screening of COVID-19. Front. Cell Infect. Microbiol. 10, 331 (2020).

52. Kitagawa, Y. et al. Evaluation of rapid diagnosis of novel coronavirus disease (COVID-19) using loop-mediated isothermal amplification. J. Clin. Virol. 129, 104446 (2020)

53. Kellner, M. J., Koob, J. G., Gootenberg, J. S., Abudayyeh, O. O. \& Zhang, F. SHERLOCK: nucleic acid detection with CRISPR nucleases. Nat. Protoc. 14, 2986-3012 (2019).

54. Chen, J. A.-O. et al. CRISPR-Cas12a target binding unleashes indiscriminate singlestranded DNase activity. Science 360, 436-439 (2018).

55. O'Connell, M. R. Molecular mechanisms of RNA targeting by Cas13-containing type VI CRISPR-Cas systems. J. Mol. Biol. 431, 66-87 (2019).

56. Gootenberg, J. S. et al. Nucleic acid detection with CRISPR-Cas13a/C2c2. Science 356, 438-442 (2017)

57. Joung, J. et al. Point-of-care testing for COVID-19 using SHERLOCK diagnostics. medRxiv https://doi.org/10.1101/2020.05.04.20091231 (2020).

58. Huang, Z. et al. Ultra-sensitive and high-throughput CRISPR-p owered COVID-19 diagnosis. Biosens. Bioelectron. 164, 112316 (2020).

59. Jeon, Y. et al. Direct observation of DNA target searching and cleavage by CRISPR-Cas12a. Nat. Commun. 9, 2777 (2018).

60. Ding, X., Yin, K., Li, Z. \& Liu, C. All-in-one dual CRISPR-Cas12a (AIOD-CRISPR) assay: a case for rapid, ultrasensitive and visual detection of novel coronavirus SARSCoV-2 and HIV virus. bioRxiv https://doi.org/10.1101/2020.03.19.998724 (2020).

61. Broughton, J. A.-O. et al. CRISPR-Cas12-based detection of SARS-CoV-2. Nat Biotechnol. 38, 870-874 (2020).

62. Myhrvold, C. A.-O. X. et al. Field-deployable viral diagnostics using CRISPR-Cas13. Science 360, 444-448 (2018).

63. Facchetti, F. et al. SARS-CoV2 vertical transmission with adverse effects on the newborn revealed through integrated immunohistochemical, electron microscopy and molecular analyses of Placenta. EBioMedicine 59, 102951 (2020).

64. Nicholls, J. M. et al. Lung pathology of fatal severe acute respiratory syndrome. Lancet 361, 1773-1778 (2003).

65. Bradley, B. T. et al. Histopathology and ultrastructural findings of fatal COVID-19 infections in Washington State: a case series. Lancet 396, 320-332 (2020).

66. Varga, Z. et al. Endothelial cell infection and endotheliitis in COVID-19. Lancet 395, 1417-1418 (2020)

67. Apolone, G. et al. Unexpected detection of SARS-CoV-2 antibodies in the prepandemic period in Italy. Tumori https://doi.org/10.1177/0300891620974755 (2020)
68. Qiu, M. et al. Antibody responses to individual proteins of SARS coronavirus and their neutralization activities. Microbes Infect. 7, 882-889 (2005).

69. Porte, L. et al. Evaluation of a novel antigen-based rapid detection test for the diagnosis of SARS-CoV-2 in respiratory samples. Int. J. Infect. Dis. 99, 328-333 (2020)

70. Diao, B. et al. Accuracy of a nucleocapsid protein antigen rapid test in the diagnosis of SARS-CoV-2 infection. Clin. Microbiol. Infect. 27, 289.e1-289.e4 (2020).

71. Ihling, C. et al. Mass spectrometric identification of SARS-CoV-2 proteins from gargle solution samples of COVID-19 patients. J. Proteome Res. 19, 4389-4392 (2020).

72. Tan, Y. J. et al. Profiles of antibody responses against severe acute respiratory syndrome coronavirus recombinant proteins and their potential use as diagnostic markers. Clin. Diagn. Lab. Immunol. 11, 362-371 (2004).

73. Kyosei, Y. et al. Proposal of de novo antigen test for COVID-19: ultrasensitive detection of Spike proteins of SARS-CoV-2. Diagnostics 10, 594 (2020).

74. Grant, B. D. et al. SARS-CoV-2 coronavirus nucleocapsid antigen-detecting halfstrip lateral flow assay toward the development of point of care tests using commercially available reagents. Anal. Chem. 92, 11305-11309 (2020).

75. Liu, D. et al. Nanozyme chemiluminescence paper test for rapid and sensitive detection of SARS-CoV-2 antigen. Biosens. Bioelectron. 173, 112817 (2020).

76. Li, H. Y. et al. Advances in detection of infectious agents by aptamer-based technologies. Emerg. Microbes Infect. 9, 1671-1681 (2020).

77. Cho, S. J., Woo, H. M., Kim, K. S., Oh, J. W. \& Jeong, Y. J. Novel system for detecting SARS coronavirus nucleocapsid protein using an ssDNA aptamer. J. Biosci. Bioeng. 112, 535-540 (2011)

78. Zhang, L. et al. Discovery of sandwich type COVID-19 nucleocapsid protein DNA aptamers. Chem. Commun. 56, 10235-10238 (2020).

79. Mak, G. C. K. et al. Evaluation of rapid antigen detection kit from the WHO emergency use list for detecting SARS-CoV-2. J. Clin. Virol. 134, 104712 (2021).

80. Mak, G. C. et al. Analytical sensitivity and clinical sensitivity of the three rapid antigen detection kits for detection of SARS-CoV-2 virus. J. Clin. Virol. 133, 104684 (2020).

81. Li, H. Y. et al. Novel aptasensor-based assay of sonic hedgehog ligand for detection of portal vein invasion of hepatocellular carcinoma. Biosens. Bioelectron. 174, 112738 (2021).

82. Mak, G. C. et al. Evaluation of rapid antigen test for detection of SARS-CoV-2 virus J. Clin. Virol. 129, 104500 (2020).

83. Scohy, A. et al. Low performance of rapid antigen detection test as frontline testing for COVID-19 diagnosis. J. Clin. Virol. 129, 104455 (2020).

84. Lisboa, B. M. et al. Diagnostic accuracy of serological tests for covid-19: systematic review and meta-analysis. BMJ 370, m2516 (2020).

85. Zainol, R. Z., Othman, S. N., Abdul, S. M. N., Ali, U. K. \& Wong, K. K. Diagnostic performance of COVID-19 serology assays. Malays. J. Pathol. 42, 13-21 (2020).

\section{ACKNOWLEDGEMENTS}

This work is supported by the National Natural Science Foundation of China (NSFC: \# 81871997 and \# 82170624 to J.W.).

\section{AUTHOR CONTRIBUTIONS}

Y.Z.: manuscript preparation; L.Z.: participation in the literature analysis and manuscript preparation; Y.X. and J.W.: overall supervision, concept development, and manuscript finalization.

\section{COMPETING INTERESTS}

The authors declare no competing interests.

\section{ETHICAL APPROVAL}

No human subjects or experimental animals were involved in this manuscript.

\section{ADDITIONAL INFORMATION}

Correspondence and requests for materials should be addressed to You-Hua Xie or Jian Wu.

Reprints and permission information is available at http://www.nature.com/ reprints

Publisher's note Springer Nature remains neutral with regard to jurisdictional claims in published maps and institutional affiliations. 